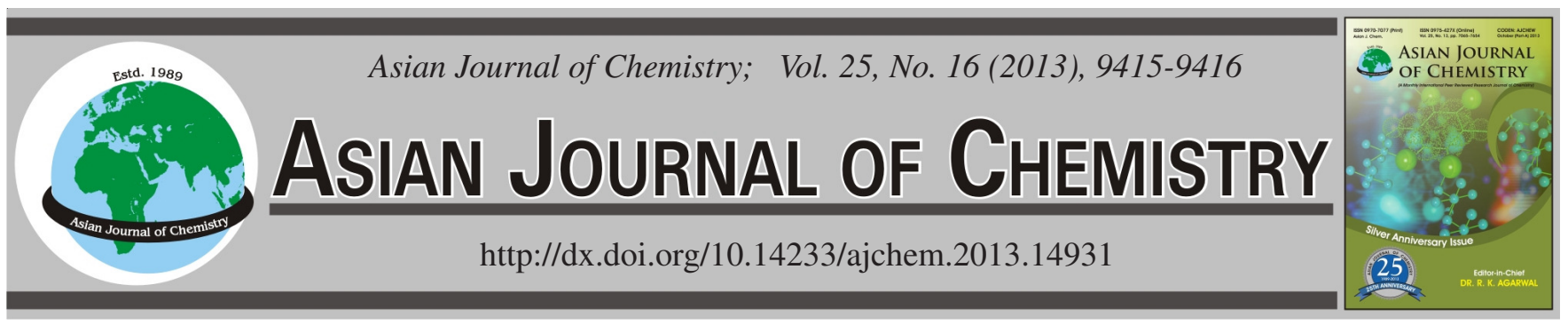

NOTE

\title{
A Practical and Efficient Preparation of Key Intermediates in the Synthesis of Praziquantel
}

\author{
Chunhua Yang, Lingzi Zhang, Yang Zheng and Dequn Sun*
}

Marine College, Shandong University, Weihai, Wenhua West Road, No.180, Weihai 264209, P.R. China

*Corresponding author: E-mail: dequn.sun@sdu.edu.cn

(Received: 8 December 2012;

Praziquantel is the first drug of choice for all worldwide people infected by schistosomiasis. Many tons of praziquantel are needed each year. In this paper, an improved process for preparation of important intermediates to manufacture the praziquantel was reported.

Key Words: Praziquantel, Intermediate, Preparation.

Schistosomiasis is a major public health problem in tropical and subtropical regions. An estimated 200 million people are infected and a total of 400 million are at risk and more than 280,000 die of schistosomiasis annually. Treatment for schistosomiasis depends almost exclusively on praziquantel (PZQ), the World Health Organization recommended drug. 100 tons of praziquantel are needed for millions of people every year.

Currently, the generic form of praziquantel is produced by several companies mainly via the process developed by Bayer. The original Bayer process ${ }^{1}$ used vast isoquinoline as starting material to yield praziquantel in a six-step synthesis. Besides the use of large amounts of benzene, a key step in the Reissert reaction using a large excess of potassium cyanide generates large volumes of cyanide waste, disposal of which is a significant environmental problem especially praziquantel is produced in several ton quantities each year. Later reports ${ }^{2-12}$ described alternate routes to synthesize praziquantel. Among these methods, an important procedure ${ }^{6}$ involving mild conditions, short steps and high overall yield $(50 \%)$ from phenyl ethylene (1) has become the most attractive method for the production of praziquantel. Amidation of chloroacetyl chloride with phenethylamine (1) in dichloromethane gave $\mathbf{2}$, which upon subsequent amination with $\mathbf{2}$ eq of aminoacetaldehyde dimethyl acetal led to $\mathbf{3}$, cyclization of $\mathbf{3}$ in large amount of $\mathrm{H}_{2} \mathrm{SO}_{4}$ afforded 4 , whose acylation with cyclohexanecarbonyl chloride gave the final product praziquantel (Scheme-I).

However, this route suffers from some limitations, such as the use of large volumes of dichloromethane in step 1, considerably low yield (67\%) and employing two equivalents of reactant aminoacetaldehyde dimethylacetal in step 2 . We

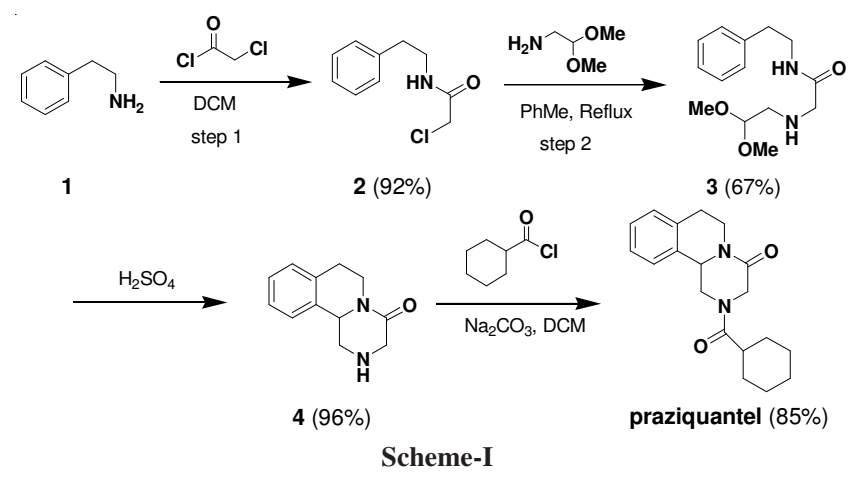

reasoned that the additional one equiv. of the aminoacetaldehyde dimethylacetal in step 2 is not effective acid scavenger to accelerate the formation of $\mathbf{3}$ efficiently, resulting in considerably lower yield compared with other steps. Our efforts were focused on improving the preparation, especially, in the preparation of intermediates $\mathbf{2}$ and $\mathbf{3}$ in high yields under mild condition (Scheme-II).

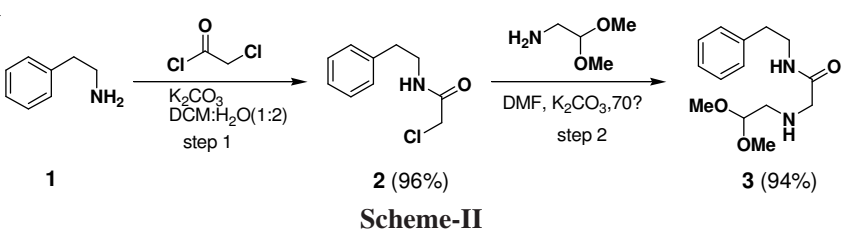

Step 1 in present method (Scheme-II) was conducted in a biphasic system composed with water and dichloromethane, which served as a more eco-friendly media and lower cost since volume of dichloromethane in this step was reduced in half compared with that in Scheme-I. Meanwhile, potassium 
carbonate was used as an acid scavenger accelerated the biphasic reaction and improved the yield over $96 \%$ in scale up preparation.

In step 2, potassium carbonate (1.2 eq) was found to be the most effective acid scavenger and also to be an attractive choice in view of (a) mild reactivity, (b) environmentally friendly by-product $\left(\mathrm{KCl}\right.$ and $\mathrm{CO}_{2}$ ), (c) low cost, (d) convenience in storage. With DMF as solvent, the 3 was obtained in $94 \%$ yield, which is much higher than $67 \%$ yield in Scheme-I.

In summary, an improved method for large-scale preparation of intermediates $\mathbf{2}$ and $\mathbf{3}$ for synthesis of praziquantel has been developed. This method will be valuable in manufacture of praziquantel by lowering production costs and reducing environmental pollution.

All commercially available materials and solvents were used without any further purification. ${ }^{1} \mathrm{H}$ NMR spectra were recorded in $\mathrm{CDCl}_{3}-d_{3}$ at $400 \mathrm{MHz}$. The chemical shift values are reported on the $\delta$ scale with respect to TMS $(\delta 0.00)$ and $\mathrm{CDCl}_{3}-d_{3}(\delta 7.26)$ as internal standards, respectively.

Scale-up preparation 2-chloro-N-phenethylacetamide (2): To a solution of phenethylamine $(1212.0 \mathrm{~g}, 10 \mathrm{~mol})$ and $\mathrm{K}_{2} \mathrm{CO}_{3}$ ( $1520.0 \mathrm{~g}, 11 \mathrm{~mol}$ ) in water $(8 \mathrm{~L})$ was added dropwise a solution of chloroacetyl chloride $(1135.0 \mathrm{~g}, 10.05 \mathrm{~mol})$ in dichloromethane $(4 \mathrm{~L})$ at room temperature. The reaction mixture was stirred with mechanical rabbling for $2 \mathrm{~h}$ at room temperature. The organic layer was separated and washed with brine. Evaporation of solvent (recovery of $\mathrm{CH}_{2} \mathrm{Cl}_{2}$ ), gave a resulting solid that was recrystallized from a mixture of $\mathrm{CH}_{3} \mathrm{OH}$ $(8 \mathrm{~L})$ and water $(6 \mathrm{~L})$ to afford $2(1901 \mathrm{~g}, 96 \%)$ as a white needles, m.p. $62-64{ }^{\circ} \mathrm{C}$ (lit. ${ }^{6}$ m.p. $\left.60-63{ }^{\circ} \mathrm{C}\right)$.
Scale-up preparation of 2-(2,2-dimethoxyethylamino)N-phenethylacetamide (3): To a mixture of 2-chloro-Nphenethylacetamide $(950 \mathrm{~g}, 4.8 \mathrm{~mol})$ and $\mathrm{K}_{2} \mathrm{CO}_{3}(731 \mathrm{~g}, 5.3$ mol) in DMF (12 L) was added aminoacetaldehyde dimethyl acetal $(556 \mathrm{~g}, 5.3 \mathrm{~mol})$ at room temperature. After stirring at $75^{\circ} \mathrm{C}$ for $5 \mathrm{~h}$, DMF was recovered by distillation and the residual yellow oil was cooled to room temperature and diluted with water $(3 \mathrm{~L})$, then extracted with dichloromethane $(1.5 \mathrm{~L}$ $\times 4)$. The combined organic solution were washed with brine and concentrated to afford $\mathbf{3}^{6}(1203 \mathrm{~g}, 94 \%)$ as a yellow oil and the dichloromethane was recovered.

\section{ACKNOWLEDGEMENTS}

The authors are grateful to National High-Tech Program of China (863 Program, 2012AA020306) for financial support.

\section{REFERENCES}

1. J. Seubert, R. Pohlke and F. Loebich, Experientia, 33, 1036 (1977).

2. D. Frehel and J.P. Maffrand, Heterocycles, 20, 1731 (1983).

3. W.F. Berkowitz and T.V. John, J. Org. Chem., 49, 5269 (1984).

4. F. Yuste, Y. Pallas, H. Barrios, B. Ortiz and R. Sanchez-Obregon, J. Heterocycl. Chem., 23, 189 (1986).

5. M.D. Brewer, M.N. Burgess, R.J.J. Dorgan, R.L. Elliott, P. Mamalis, B.R. Manger and R.A. Webster, J. Med. Chem., 32, 2058 (1989).

6. J.H. Kim, Y.S. Lee, H. Park and C.S. Kim, Tetrahedron, 54, 7395 (1998).

7. J.H. Kim, Y.S. Lee and C.S. Kim, Heteroeycles, 48, 2279 (1998).

8. M.R.C.G. Novaes, J.P. de Souza and H.C. de Araujo, Quim Nova, 22, 5 (1999); Chem. Abstr., 130, 196623 (1999).

9. M.H. Todd, C. Ndubaku and P.A. Bartlett, J. Org. Chem., 67, 3985 (2002).

10. C. Ma, Q.F. Zhang, Y.B. Tan and L. Wang, J. Chem. Res., 3, 186 (2004).

11. S. E1-Fayyoumy, W. Mansour and M.H. Todd, Tetrahedron Lett., 47, 1287 (2006).

12. H.P. Cao, H.X. Liu and A. Domling, Chem. Eur. J., 16, 12296 (2010). 